\title{
Particle Filters for State and Parameter Estimation in Batch Processes
}

\author{
Tao Chen, Julian Morris and Elaine Martin \\ Centre for Process Analytics and Control Technology, \\ School of Chemical Engineering and Advanced Materials, \\ University of Newcastle upon Tyne, \\ Newcastle upon Tyne, NE1 7RU, U.K. \\ e-mail: e.b.martin@ncl.ac.uk; Tel. No.+44 191222 6231; Fax No. +44 1912225748
}

\begin{abstract}
In process engineering, on-line state and parameter estimation is a key component in the modelling of batch processes. However, when state and/or measurement functions are highly non-linear and the posterior probability of the state is non-Gaussian, conventional filters, such as the extended Kalman filter, do not provide satisfactory results. This paper proposes an alternative approach whereby particle filters based on the sequential Monte Carlo method are used for the estimation task. Particle filters are initially described prior to discussing some implementation issues, including degeneracy, the selection of the importance density and the number of particles. A kernel smoothing approach is introduced for the robust estimation of unknown and time-varying model parameters. The effectiveness of particle filters is demonstrated through application to a benchmark batch polymerization process and the results are compared with the extended Kalman filter.
\end{abstract}

Key words: Batch processes; Parameter estimation; Particle filters; Sequential Monte Carlo; State estimation. 


\section{Introduction}

Developments in process engineering have resulted in the need for improved methodologies that will realise the more detailed modelling of chemical production processes, thereby enabling the implementation of enhanced process optimisation, monitoring and control schemes. A number of different approaches to process modelling exist, phenomenological (mechanistic), empirical and hybrid (a combination of mechanistic and data-based approaches). Of particular interest in this paper is mechanistic modelling where the process of interest is described through a set of differential equations that are usually discretized to a set of difference equations. These equations can be regarded as a general state space model [1], with the difference variables defining the states. The term "state space model" is also widely referred to in data driven modelling [2] where the states and model are identified using process operational data. In this paper, unless otherwise stated, a "state space model" is a discretized mechanistic model. Critical to the real-time deployment of a mechanistic model is the on-line estimation of both the states and the parameters from data collected from the actual process. However a number of issues arise as a consequence of the fact that some state variables cannot be measured directly on-line (e.g. polymer properties in a batch polymerization process [3]). In addition, some of the parameters in a state space model cannot be specified exactly a priori $[3,4,5]$. These issues are discussed and addressed in this paper.

State estimation can be considered as an optimal filtering problem within a Bayesian framework. If the state equations are linear and the posterior density, at every time step, is Gaussian, the Kalman filter $(\mathrm{KF})[1]$ is an optimal solution to the state estimation problem. However, when these assumptions do not hold, there exists no analytical solution and therefore approximations have to be made. For example the extended Kalman filter (EKF) has been widely applied to estimate nonlinear state space models [3,6,7]. The EKF assumes a Gaussian posterior density and adopts a firstorder Taylor series expansion to provide a local approximation to the current state. However when state equations are highly non-linear and the posterior density is non-Gaussian, the EKF may give a high estimation error. To avoid the Gaussian assumption, one approach that was originally presented in the 1970's was to approximate the posterior density by discretizing the continuous state variables into grids $[5,8,9]$. This methodology was termed point-mass filters or probability-grid filters. However the computational cost of point-mass filters increases exponentially with the state dimension, thus limiting its widespread application, at the current time, in process engineering.

This paper introduces particle filters for dynamic state and parameter estimation [10, 11]. Particle filters are an extension to point-mass filters. The basic idea is that a large number of samples 
(particles) are generated using Monte Carlo methods to approximate the posterior probability of the states. Therefore the particles are adaptively concentrated in regions of high probability. This is in contrast to point-mass filters which adopt a pre-defined discretization approach to the state space problem, resulting in the particles being assumed to be uniformly distributed over the space. During the past decade, particle filters have developed rapidly and have been successfully applied in a number of different areas [11]. There has, however, been limited application of particle filters in process engineering. Examples include the state estimation of a non-linear dynamic process [12], and state estimation with initial condition rectification, which was implemented using a Markov chain Monte Carlo approach [13].

The extension of particle filters to parameter estimation is non-trivial. The conventional strategy is to add a random walk to the parameters, and then augment the state space with the parameters for the joint estimation. However the use of a random walk implies an increase in the covariance of the parameters, resulting in posteriors more diffuse than the actual ones. Kernel smoothing [14] is proposed to rectify the covariance issue, by determining the covariance of parameters based on particles from previous time points. Kernel smoothing is a flexible approach to handling both fixed and time-varying parameters.

The particle filters are demonstrated by application to a simulated benchmark batch polymerization reactor for state and parameter estimation, and are observed to exhibit improved performance over the extended Kalman filter for the estimation of the polymer properties.

\section{Particle Filters for State Space Estimation}

The following description of particle filters is based on the tutorial of Arulampalam et al. [10]. A general dynamic state space estimation problem is first described through a Bayesian framework. Particle filters are then introduced as approximate numerical solutions. This is followed by a discussion of a number of implementation issues including degeneracy, the selection of the importance density and the number of particles. By addressing these issues, an efficient particle filter algorithm is developed.

\subsection{Bayesian Estimation of State Space Model}

Consider the following state space model with non-linear state and measurement functions, $\mathbf{f}_{k}$ and $\mathbf{h}_{k}$ respectively: 
$\mathbf{x}_{k}=\mathbf{f}_{k}\left(\mathbf{x}_{k-1}, \mathbf{v}_{k-1}\right)$

$\mathbf{z}_{k}=\mathbf{h}_{k}\left(\mathbf{x}_{k}, \mathbf{n}_{k}\right)$

$k$ is the time index, $\mathbf{x}$ is a state vector, and $\mathbf{z}$ is the measurement vector. $\mathbf{v}$ and $\mathbf{n}$ are independent and identically distributed noise for the process and measurements, respectively. The objective of state estimation is to sequentially calculate the state vector, $\mathbf{x}_{k}$, given the measurements $\mathbf{z}_{k}$. In real processes, some states are difficult to measure on-line, such as the molecular weight of polymers and the concentration of reactant, with some being unmeasurable. Therefore one of the challenges in state estimation is to infer all the states from limited measurements.

From a Bayesian perspective, the aim of state estimation is to infer the probability function of the state $\mathbf{x}_{k}$ given the measurement sequence $\mathbf{z}_{1: k}\left(\mathbf{z}_{1: k}=\left\{\mathbf{z}_{1}, \mathbf{z}_{2}, \ldots, \mathbf{z}_{k}\right\}\right)$, i.e., $p\left(\mathbf{x}_{k} \mid \mathbf{z}_{1: k}\right)$. Assuming the initial conditions, expressed in the form of a probability distribution function $p\left(\mathbf{x}_{0} \mid \mathbf{z}_{0}\right) \equiv p\left(\mathbf{x}_{0}\right)$, is available, $p\left(\mathbf{x}_{k} \mid \mathbf{z}_{1: k}\right)$ can be obtained sequentially through prediction:

$p\left(\mathbf{x}_{k} \mid \mathbf{z}_{1: k-1}\right)=\int p\left(\mathbf{x}_{k} \mid \mathbf{x}_{k-1}\right) p\left(\mathbf{x}_{k-1} \mid \mathbf{z}_{1: k-1}\right) d \mathbf{x}_{k-1}$

and updated as follows:

$p\left(\mathbf{x}_{k} \mid \mathbf{z}_{1: k}\right)=\frac{p\left(\mathbf{z}_{k} \mid \mathbf{x}_{k}\right) p\left(\mathbf{x}_{k} \mid \mathbf{z}_{1: k-1}\right)}{p\left(\mathbf{z}_{k} \mid \mathbf{z}_{1: k-1}\right)}$

$p\left(\mathbf{z}_{k} \mid \mathbf{z}_{1: k-1}\right)$ is a normalizing factor independent of the state $\mathbf{x}_{k}$.

In equations (3) and (4), $p\left(\mathbf{x}_{k} \mid \mathbf{x}_{k-1}\right)$ is defined by the state function, equation (1) and $p\left(\mathbf{z}_{k} \mid \mathbf{x}_{k}\right)$ by the measurement function, equation (2). Similar probability functions for smoothing $p\left(\mathbf{x}_{k-s} \mid \mathbf{z}_{1: k}\right), \quad s>0$ and prediction, $p\left(\mathbf{x}_{k+s} \mid \mathbf{z}_{1: k}\right), \quad s>0$, can be defined. However this paper is only concerned with filtering, and thus the only situation of interest is where $s=0$. Equations (3) and (4) are the optimal solution from a Bayesian perspective to the non-linear state estimation 
problem. In general, the posterior probability, $p\left(\mathbf{x}_{k} \mid \mathbf{z}_{1: k}\right)$, cannot be determined analytically. Thus approximate filters are used to provide suboptimal solutions. The widely used EKF may work poorly for highly non-linear systems because of the Taylor approximation. In addition, even if $p\left(\mathbf{x}_{k-1} \mid \mathbf{z}_{k-1}\right)$ is Gaussian, $p\left(\mathbf{x}_{k} \mid \mathbf{z}_{k}\right)$ is no longer Gaussian due to the non-linear state function, which invalidates the underlying assumption of the EKF. An alternative approach is through particle filters.

\subsection{Overview of Particle Filters}

The basic idea of particle filters is to approximate $p\left(\mathbf{x}_{k} \mid \mathbf{z}_{1: k}\right)$ using a set of random samples (also called particles) $\left\{\mathbf{x}_{k}^{i}, i=1, \ldots, N\right\}$ with associated weights $\left\{w_{k}^{i}, i=1, \ldots, N\right\}$ where $\sum_{i=1}^{N} w_{k}^{i}=1$ :

$p\left(\mathbf{x}_{k} \mid \mathbf{z}_{1: k}\right) \approx \sum_{i=1}^{N} w_{k}^{i} \delta\left(\mathbf{x}_{k}-\mathbf{x}_{k}^{i}\right)$

$\delta(\mathbf{x})$ is an indicator function which is equal to unity if $\mathbf{x}=\mathbf{0}$; otherwise it is equal to zero. Therefore the key step is to generate random samples from $p\left(\mathbf{x}_{k} \mid \mathbf{z}_{1: k}\right)$. However, as $p\left(\mathbf{x}_{k} \mid \mathbf{z}_{1: k}\right)$ is not of the conventional form of a probability density function, such as Gaussian or Cauchy, direct sampling is not possible. Therefore importance sampling [15] is used to obtain the particles and associated weights. The first step in importance sampling is to define an importance density $q\left(\mathbf{x}_{k} \mid \mathbf{z}_{1: k}\right)$, from which samples $\mathbf{x}_{k}^{i}$ can be drawn (e.g. a standard Gaussian distribution function). Thus the weights are defined as:

$$
w_{k}^{i} \propto \frac{p\left(\mathbf{x}_{k}^{i} \mid \mathbf{z}_{1: k}\right)}{q\left(\mathbf{x}_{k}^{i} \mid \mathbf{z}_{1: k}\right)}
$$

For the sequential estimation problem, at time point $k$, the particles which approximate $p\left(\mathbf{x}_{k-1} \mid \mathbf{z}_{1: k-1}\right)$ will be passed through the state function and updated with a new measurement, $\mathbf{z}_{k}$ to approximate $p\left(\mathbf{x}_{k} \mid \mathbf{z}_{1: k}\right)$. It was shown in [10] that if the importance density is only dependent on the current measurement, $\mathbf{z}_{k}$, and the past state, $\mathbf{x}_{k-1}$, the weights can be updated as: 
$w_{k}^{i} \propto w_{k-1}^{i} \frac{p\left(\mathbf{z}_{k} \mid \mathbf{x}_{k}^{i}\right) p\left(\mathbf{x}_{k}^{i} \mid \mathbf{x}_{k-1}^{i}\right)}{q\left(\mathbf{x}_{k}^{i} \mid \mathbf{x}_{k-1}^{i}, \mathbf{z}_{k}\right)}$

With these particles and associated weights, the estimated state vector, $\hat{\mathbf{x}}_{k}$, is the mean of $p\left(\mathbf{x}_{k} \mid \mathbf{z}_{1: k}\right)$ and is calculated as:

$\hat{\mathbf{x}}_{k}=\sum_{i=1}^{N} w_{k}^{i} \mathbf{x}_{k}^{i}$

\subsection{Implementation Issues}

Several implementation issues are considered, including degeneracy, the selection of the importance density, and the number of particles required. Degeneracy is where, after a number of time steps, only one particle has significant weight. Doucet [16] showed that the variance of the importance weights increases over time, making degeneracy unavoidable. Thus considerable computational effort is expended on updating particles whose contribution to the approximation of $p\left(\mathbf{x}_{k} \mid \mathbf{z}_{1: k}\right)$ is negligible. Re-sampling [17], which is a variant of the bootstrap technique, can be used to eliminate those particles with small weights, thereby focussing the analysis on particles with large weights. A new particle set is thus generated by sampling with replacement from the original set $\left\{\mathbf{x}_{k}^{i}, i=1, \ldots, N\right\}$ with probability $\operatorname{Pr}\left(\mathbf{x}_{k}^{j}=\mathbf{x}_{k}^{i}\right)=w_{k}^{i}$. Here $j$ is the particle index after re-sampling. The "updated relationship" is denoted as $\operatorname{parent}(j)=i$. The weights are re-set to $1 / N$ as the particles are independent and identically distributed and drawn from a discrete density function.

The second issue is how to select the importance density. One approach is to use the prior distribution $p\left(\mathbf{x}_{k} \mid \mathbf{x}_{k-1}^{i}\right)$, to yield a simple form for updating the weights:

$w_{k}^{i} \propto w_{k-1}^{i} p\left(\mathbf{z}_{k} \mid \mathbf{x}_{k}^{i}\right)$

However, as this importance density is independent of the current measurement, the state space is explored without knowledge of the measurements, which makes the filter sensitive to outliers. A more reliable importance density was proposed in [18]: 
$q\left(\mathbf{x}_{k}^{i} \mid \mathbf{z}_{k}\right) \propto p\left(\mathbf{z}_{k} \mid \boldsymbol{\mu}_{k}^{i}\right) p\left(\mathbf{x}_{k} \mid \mathbf{x}_{k-1}^{i}\right) w_{k-1}^{i}$

where $\boldsymbol{\mu}_{k}^{i}$ is defined in this paper as the mean of $p\left(\mathbf{x}_{k} \mid \mathbf{x}_{k-1}^{i}\right)$. The particle index, $i$, also serves as an auxiliary variable for deriving the importance density. By utilising $\boldsymbol{\mu}_{k}^{i}$, new particles are generated from particles at the previous time step, conditional on the current measurement $\mathbf{z}_{k}$, which will be closer to the true states. In addition from Bayes's rule:

$p\left(\mathbf{x}_{k}^{i} \mid \mathbf{z}_{k}\right) \propto p\left(\mathbf{z}_{k} \mid \mathbf{x}_{k}\right) p\left(\mathbf{x}_{k} \mid \mathbf{x}_{k-1}^{i}\right) w_{k-1}^{i}$

Considering the re-sampling stage, the particle $\mathbf{x}_{k}^{j}$ is assigned a weight proportional to the ratio of the right-hand side of equations (11) and (10):

$w_{k}^{j} \propto \frac{p\left(\mathbf{z}_{k} \mid \mathbf{x}_{k}^{j}\right)}{p\left(\mathbf{z}_{k} \mid \boldsymbol{\mu}_{k}^{\operatorname{parent}(j)}\right)}$

Filters with this importance density and re-sampling stage are termed Auxiliary Sampling Importance Re-sampling (ASIR) filters. In summary, the ASIR algorithm for the current time point $k$ is as follows:

(1) For $i=1: N$

$$
\begin{aligned}
& \text { Calculate } \boldsymbol{\mu}_{k}^{i} \\
& \text { Calculate } w_{k}^{i} \propto p\left(\mathbf{z}_{k} \mid \boldsymbol{\mu}_{k}^{i}\right) w_{k-1}^{i}
\end{aligned}
$$

End

(2) Normalize $w_{k}^{i}$ such that they sum to 1.

(3) Re-sample.

(4) For $j=1: N$

Draw $\mathbf{x}_{k}^{j}$ from $p\left(\mathbf{x} \mid \mathbf{x}_{k-1}^{\operatorname{parent}(j)}\right)$

Assign the weights using equation (12)

End 
(5) Normalize $w_{k}^{j}$ such that they sum to 1.

Finally, the number of particles required is not only dependent on the state dimension, but also on the specific distribution of the states. If the states are independent of each other, the number of required particles increases exponentially with the state dimension, similar to point-mass filters. However, in practical problems, states tend be correlated and thus the joint distribution tends to concentrate along some "curve", implying lower dimensionality than for independent states. Therefore fewer particles are sufficient to approximate the joint distribution. In practice, the number of particles is usually decided empirically by some initial experiments.

\section{Kernel Smoothing}

If both states and model parameters are to be estimated, Bayes's rule gives the following joint posterior distribution:

$$
p\left(\mathbf{x}_{k}, \boldsymbol{\theta}_{k} \mid \mathbf{z}_{1: k}\right) \propto p\left(\mathbf{z}_{k} \mid \mathbf{x}_{k}, \boldsymbol{\theta}_{k}\right) p\left(\mathbf{x}_{k} \mid \boldsymbol{\theta}_{k}, \mathbf{z}_{1: k-1}\right) p\left(\boldsymbol{\theta}_{k} \mid \mathbf{z}_{1: k-1}\right)
$$

where $\boldsymbol{\theta}_{k}$ is the vector of model parameters. Joint state and parameter estimation is achieved through the augmentation of the state space with the parameter vector. A Gaussian random walk for the parameters can then be specified to enable their adaptation to new data:

$\boldsymbol{\theta}_{k}=\boldsymbol{\theta}_{k-1}+\zeta_{k}$

where $\zeta_{k} \sim G\left(\mathbf{0}, \mathbf{W}_{k}\right)$ satisfies a Gaussian distribution with zero mean and some pre-defined covariance matrix, $\mathbf{W}_{k}$. This strategy has been widely adopted in conventional state and parameter estimation techniques, such as the EKF [3]. However, as identified by some researchers [14,19], the random walk implies an increase in the covariance, resulting in posteriors more diffuse than the actual ones. This issue can be demonstrated in the framework of particle filters, where $p\left(\boldsymbol{\theta}_{k} \mid \mathbf{z}_{1: k-1}\right)$ is approximated by a mixture of particles:

$$
p\left(\boldsymbol{\theta}_{k} \mid \mathbf{z}_{1: k-1}\right) \approx \sum_{i=1}^{N} w_{k-1}^{i} G\left(\boldsymbol{\theta}_{k} \mid \boldsymbol{\theta}_{k-1}^{i}, \mathbf{W}_{k}\right)
$$


Let $\overline{\boldsymbol{\theta}}_{k-1}$ and $\mathbf{V}_{k-1}$ be the Monte Carlo mean and covariance matrices computed from all the particles with weights, $\left\{\boldsymbol{\theta}_{k-1}^{i}, w_{k-1}^{i}, i=1, \ldots, N\right\}$. It is noted in [14] that the distribution in equation (15) has a mean of $\overline{\boldsymbol{\theta}}_{k-1}$ and covariance matrix $\mathbf{V}_{k-1}+\mathbf{W}_{k}$. As a consequence of the covariance of the random walk $\left(\mathbf{W}_{k}\right)$, the covariance increases over time. A natural approach to reducing the covariance is to use kernel smoothing [14] with smoothing factor, $1>h>0$ :

$p\left(\boldsymbol{\theta}_{k} \mid \mathbf{z}_{1: k-1}\right) \approx \sum_{i=1}^{N} w_{k-1}^{i} G\left(\boldsymbol{\theta}_{k} \mid \mathbf{m}_{k-1}^{i}, h^{2} \mathbf{V}_{k-1}\right)$

The kernel locations $\mathbf{m}_{k-1}^{i}$ are specified by a shrinkage rule that forces the particles to be closer to their mean:

$\mathbf{m}_{k-1}^{i}=\left(\sqrt{1-h^{2}}\right) \boldsymbol{\theta}_{k-1}^{i}+\left(1-\sqrt{1-h^{2}}\right) \overline{\boldsymbol{\theta}}_{k-1}$

It is straightforward to verify that the mixture probability in equation (16) has a mean of $\overline{\boldsymbol{\theta}}_{k-1}$ and covariance matrix $\mathbf{V}_{k-1}$, and that the covariance matrix does not increase over time [14]. Thus, at each time step, $k$, particles for $\boldsymbol{\theta}_{k}$ are drawn from the mixture density. Incorporating this step into the ASIR filter, the combined state and parameter estimation algorithm for the current time point $k$ is as follows.

(1) For $i=1: N$

Calculate $\boldsymbol{\mu}_{k}^{i}$ and $\mathbf{m}_{k-1}^{i}$

Calculate $w_{k}^{i} \propto p\left(\mathbf{z}_{k} \mid \boldsymbol{\mu}_{k}^{i}, \mathbf{m}_{k-1}^{i}\right) w_{k-1}^{i}$

End

(2) Normalize $w_{k}^{i}$ such that they sum to 1 .

(3) Re-sample.

(4) For $j=1: N$

Draw $\boldsymbol{\theta}_{k}^{j}$ from Gaussian distribution $G\left(. \mid \mathbf{m}_{k-1}^{\operatorname{parent}(j)}, h^{2} \mathbf{V}_{k-1}\right)$ 
Draw $\mathbf{x}_{k}^{j}$ from $p\left(\mathbf{x}_{k} \mid \mathbf{x}_{k-1}^{\text {parent }(j)}, \boldsymbol{\theta}_{k}^{j}\right)$

Assign weight $w_{k}^{j}$ according to

$$
w_{k}^{j} \propto \frac{p\left(\mathbf{z}_{k} \mid \mathbf{x}_{k}^{j}, \boldsymbol{\theta}_{k}^{j}\right)}{p\left(\mathbf{z}_{k} \mid \boldsymbol{\mu}_{k}^{\text {parent }(j)}, \mathbf{m}_{k-1}^{\text {parent }(j)}\right)}
$$

End

(5) Normalize $w_{k}^{j}$ such that they sum to 1.

The kernel smoothing algorithm provides a flexible approach to dealing with unknown parameters. If the parameters are known a priori to be fixed or slowly-varying, the smoothing factor $h$ should be set to a small positive value (e.g. $0<h<0.2)$ to reflect its (nearly) steady property. On the other hand, if the parameters are expected to change significantly over time, $h$ should take a value close to 1.0 (e.g. $0.8<h<1$ ) to incorporate the inherent dynamic characteristics of the process. In practice, this factor is tuned on a validation data-set, and then applied to future batches.

\section{Simulation Results}

In this section, the particle filtering technique is evaluated through its application to a simulation of a benchmark batch polymerization process. The results of the EKF are presented for comparison.

\subsection{Polymerization Process Model}

The process shown in Figure 1 is a free-radical batch polymerization of methyl-methacrylate (MMA) with a water solvent and benzoyl peroxide initiator [3,4,20]. The polymerization temperature is maintained by a cascade control system through manipulating the flow rates of the hot and cold water streams. A detailed mathematical model of the process is described in [20]. A simulation program has been developed [4] and is used as a test bed for the evaluation of particle filters for state and parameter estimation. The detailed settings for the simulation, such as process kinetic parameters, physical properties, reactor operating conditions, and controller configurations, can be found in [3]. The system comprises 11 state variables (Table 1) that provide the minimum information required to calculate the polymer properties of interest, including number average molecular weight $(\mathrm{Mn})$ and weight average molecular weight $(M w)$. These properties are related to the leading moments of the deactivated polymer chains. 
In particle filters, instead of simply calculating the polymer properties using the mean of the states, a Monte Carlo approximation approach is adopted which is the weighted average over the particles:

$$
\begin{aligned}
& M n=100.05 \times \frac{\mu_{1}-\lambda_{1}}{\mu_{0}-\lambda_{0}} \approx 100.05 \times \frac{\mu_{1}}{\mu_{0}} \approx \sum_{i=1}^{N} 100.05 \times \frac{\mu_{1}^{i}}{\mu_{0}^{i}} \times w^{i} \\
& M w=100.05 \times \frac{\mu_{2}-\lambda_{2}}{\mu_{1}-\lambda_{1}} \approx 100.05 \times \frac{\mu_{2}}{\mu_{1}} \approx \sum_{i=1}^{N} 100.05 \times \frac{\mu_{2}^{i}}{\mu_{1}^{i}} \times w^{i}
\end{aligned}
$$

where the superscript $i$ denotes the particle index, $\left\{\lambda_{j}, j=0,1,2\right\}$ are the $j$-th moments of live polymer, and $\left\{\mu_{j}, j=0,1,2\right\}$ are the $j$-th moments of dead polymer. In this study, it is assumed that on-line measurements are available for monomer conversion, and the measured reactor and jacket temperature, which are corrupted by white Gaussian noise with signal-to-noise ratio, $20 \mathrm{db}$. The measurement interval is 1 minute with batch duration being 120 minutes. The root mean square error (RMSE) of the estimated polymer properties forms the basis of the performance evaluation. The ASIR filter with kernel smoothing is implemented for the state and parameter estimation task. To further investigate the robustness of the ASIR filter to random perturbations, 100 realizations of the process were generated by adding measurement noise from different random seeds. Thus the uncertainty of estimation performance is assessed using the results from the 100 realizations.

\subsection{State Estimation with Incorrect Prior for the Initial Initiator Concentration}

The polymer molecular properties can be significantly affected by unknown amounts of reactive impurities in the monomer and/or solvent feed streams. Therefore, estimation of initial initiator concentration is essential for on-line state estimation. To undertake a fair comparison both the EKF and ASIR filter were implemented in recursive form, consequently all information from previous time steps is included in the calculation of the current estimates. If explicit use of the information from the previous time points is possible in practice, MCMC can be incorporated into the ASIR filter [13] thereby making use of information from a time-horizon. However for the EKF, an optimisation-based batch-wise estimator is normally adopted [21].

In the simulated MMA batch process, the initial initiator concentration is assumed to be $20 \%$ lower than its nominal value because of the presence of reactive impurities. One batch is randomly selected from the 100 simulated batches to tune the covariance matrices (process noise, 
measurement noise, and initial states) for the EKF and particle filters, and to attain the kernel smoothing factor $h$. Considering the initial initiator concentration as a fixed parameter, the tuning interval for $h$ was set to $(0,0.2)$, with a resolution of 0.02 . The tuning parameters which give the best results will be applied to the estimation task for the remaining 99 batches.

The results in Table 2 show that with an incorrect initial estimate of the initial initiator concentration in the MMA model, the particle filter can achieve significantly more accurate estimates of the polymer properties than the EKF. The 95\% confidence bounds for the RMSE, based on 100 realizations, also indicates that the ASIR filter is more reliable and robust than the EKF. Figure 2 and Figure 3 show the results for one simulation for the on-line estimation of number average molecular weight and weight average molecular weight, respectively. These results are selected for presentation, since they are representative of all the simulations, with the RMSE for both the EKF and particle filters being close to the mean values. This criterion was subsequently used to select the figures in Section 4.3. It can be seen that in both Figures 2 and 3, the ASIR filter follows the true process trajectory more closely than the EKF. The results for initiator concentration in Figure 4 clearly indicate that the ASIR filter converges to the actual value after about 20 minutes. On the other hand, the EKF's slow convergence results in poorer performance in estimating the polymer properties. The faster convergence of the particle filter is potentially due to two important points: 1) the approximation using particles is more accurate than the Gaussian assumption in the EKF, and 2) kernel smoothing determines the covariance of the parameters based on particles from the previous time point, in contrast to the pre-tuned values in the EKF.

The CPU time is summarised in Table 2. These results were obtained on a Pentium-4 $3.0 \mathrm{GHz}$ computer running under Windows XP. The computational cost of the ASIR filter increases approximately linearly with the number of particles. As the whole batch process runs for 120 minutes, the ASIR filter can satisfy the necessary on-line filtering requirements in terms of providing accurate estimates early in the batch trajectory. This is particularly important where online, real-time trajectory optimisation is required.

The other issue with the particle filter is to decide on the number of particles. The appropriate number of particles is normally determined by the state dimensions, the non-linearity of the system, and the properties of the unknown parameters. In general more particles achieve better performance i.e. a lower RMSE with tighter confidence bounds. Intuitively, 200 particles for the 12 dimensional problem would mean that fewer than 2 particles $(\sqrt[12]{200} \approx 1.555<2)$ are used for each independent 
axis. However for this specific system, dependency exists among the states. For example one would expect a high correlation between reactor temperature and jacket temperature, and among the three leading moments of dead polymer. Therefore the latent independent dimension of the states is lower, and a reasonable number of particles are required. In this example, 1000 particles provide marginally lower estimation error than 200 particles, implying 200 particles are sufficient to obtain reliable results.

\subsection{State Estimation with Time-Varying Kinetic Parameter}

In real chemical processes, model parameters can vary over time. The following study considers an unknown, time-varying kinetic parameter, the termination rate constant $\left(k_{t}\right)$. Because of the gel effect, the termination rate constant can vary with monomer conversion in free-radical polymerization. In general $k_{t}$ can be represented by an inherent chemical rate constant, $k_{t 0}$, and a diffusion-controlled function $g_{t}$, accounting for the observed decrease of $k_{t}$. In addition, to take

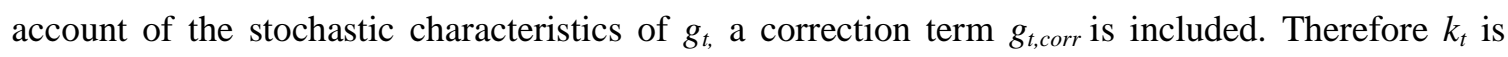
expressed as a product of these three terms: $k_{t}=k_{t 0} g_{t} g_{t, \text { corr }} . g_{t, \text { corr }}=1.0$ implies that no plant-model mismatch exists.

To simulate the time-varying effect, the actual value of $g_{t, \text { corr }}$ is assumed to follow a linear decreasing trend with random noise:

$g_{t, c o r r, k}=g_{t, c o r r, 0}-\frac{0.25 k}{120}+v$,

where $k$ is the time index in minutes. $v$ is Gaussian noise with zero mean and a standard deviation of 0.01 . The initial estimate for $g_{t, \text { corr }}$ is set to the nominal value of 1.0 , whilst the true value is 0.9 . $g_{t, c o r r}$ is assumed to follow a Gaussian random walk for the EKF. Kernel smoothing, as described in Section 3, is adopted to estimate $g_{t, c o r r}$. A randomly selected batch is used to tune the parameters of the EKF and ASIR filter. As the termination rate constant is known, a priori, to be time-varying, the tuning interval for the kernel smoothing factor $h$ is set to $(0.8,1.0)$ with a resolution of 0.02 . In addition, these tuning experiments also indicated that 200 particles were not sufficient to address the varying behaviour of the kinetic parameter. Therefore 1000 particles were used and the results are shown in Table 3. 
For this study, the ASIR filter outperformed the EKF in terms of inferring the polymer properties. The RMSE in Table 3 confirms this conclusion. Figure 5 and Figure 6 illustrate the estimated polymer properties against the true values, with superior results being attained for the ASIR filter. At the beginning of the batch, the oscillations present in the particle filters are due to the large initial covariance for the states, which allows the methodology to search over large regions for areas of high probability for the states, resulting in the fast tracking of the polymer properties. However in the tuning procedure, an EKF with a large initial covariance will diverge.

The other interesting finding is that both the particle filter and the EKF cannot obtain satisfactory estimates of the termination rate constant, with the results for the particle filters being slightly poorer than those of the EKF. However the particle filter follows the trend of the termination rate constant more closely with an approximately constant offset, up to the impact of the gel effect, whilst although the EKF generally has smaller errors is not consistent in terms of tracking the trajectory of the termination rate constant. This issue is compounded by the fact, that following an investigation of the process indicates, that the available measurements (monomer conversion and temperatures) do not provide sufficient information to estimate the termination rate constant accurately. However, without linearization and the Gaussian assumption as for the EKF, the particle filters are shown to be more robust to the error in the parameter estimates, giving significantly better results in terms of the prediction of the polymer properties.

\section{Conclusions}

This paper introduced the application of particle filters for on-line state and parameter estimation in a highly non-linear batch process. Without assuming a linear or Gaussian system, particle filters were observed to be suitable for dealing with a general state space model. An efficient particle filter algorithm, auxiliary sampling importance re-sampling (ASIR) filter, was described for the state estimation task. With respect to the unknown parameters, a kernel smoothing method was incorporated into the particle filter to obtain robust estimates for both the fixed and time-varying parameters. The particle filtering framework was evaluated on a simulated benchmark batch polymerization reactor and promising results were achieved.

The implications of this study is that particle filters are particularly attractive for applications requiring on-line state estimation of mechanistic models, such as model based quality monitoring, predictive control and data rectification. An extension of the research to an industrial batch 
crystallization process is ongoing. Further improvements of the importance density for particle filters is also under investigation.

\section{Acknowledgments}

T. Chen would like to acknowledge the financial support of the EPSRC award KNOW-HOW (GR/R19366/01) and Chemicals Behaving Badly II award (GR/R43853/01), and the UK ORS Award for his $\mathrm{PhD}$ study.

\section{References}

1. A. H. Jazwinski, Stochastic Processes and Filtering Theory, Academic, New York, 1970.

2. C. D. Schaper, W. E. Larimore, D. E. Seborg, D. A. Mellichamp, Identification of Chemical Processes using Canonical Variate Analysis, Computers and Chemical Engineering, 18 (1994) $55-69$.

3. C. Kiparissides, P. Seferlis, G. Mourikas, A. J. Morris, On-line Optimizing Control of Molecular Weight Properties in Batch Free-Radical Polymerization Reactors, Industrial and Engineering Chemistry Research, 41 (2002) 6120-6131.

4. G. Mourikas, Modelling, Estimation and Optimisation of Polymerisation Processes, Ph.D. Thesis, University of Newcastle, UK (1998).

5. P. Terwiesch, M. Agarwal, A Discretized Non-linear State Estimator for Batch Processes, Computers and Chemical Engineering, 19 (1995) 155-169.

6. D. J. Kozub, J. F. MacGregor, State Estimation for Semibatch Polymerization Reactors, Chemical Engineering Science, 47 (1992) 1047-1062.

7. N. L. Ricker, J. H. Lee, Non-linear Modeling and State Estimation for the Tennessee-Eastman Challenge Process, Computers and Chemical Engineering, 19 (1995) 983-1005.

8. R. S. Bucy and K. D. Senne, Digital Synthesis of Non-linear Filters, Automatica, 7 (1971) 287298

9. H. W. Sorenson, Recursive Estimation for Non-linear Dynamic Systems, in J. C. Spall (Eds.), Bayesian Analysis of Time Series and Dynamic Models, Marcel Dekker, New York, 1988, 127165.

10. M. Arulampalam, S. Maskell, N. Gordon, T. Clapp, A Tutorial on Particle Filters for On-line Non-linear/Non-gaussian Bayesian Tracking, IEEE Transactions on Signal Processing, 50 (2002) 174-188.

11. A. Doucet, N. de Freitas, N. Gordon (Eds.), Sequential Monte Carlo in Practice, SpringerVerlag, New York, 2001. 
12. W.-S. Chen, B. R. Bakshi, P. K. Goel, S. Ungarala, Bayesian Estimation of Unconstrained Nonlinear Dynamic Systems, Proc. of International Symposium on Advanced Control of Chemical Processes, 2004.

13. T. Chen, J. Morris, E. Martin, Particle Filters for the Estimation of a State Space Model, Proc. of European Symposium on Computer Aided Process Engineering, 2004.

14. J. Liu, M. West, Combined Parameter and State Estimation in Simulation-based Filtering, in A. Doucet, N. de Freitas, N. Gordon (Eds.), Sequential Monte Carlo in Practice, Springer-Verlag, New York, 2001, 197-223.

15. C. Robert, G. Casella, Monte Carlo Statistical Methods, Springer, New York, 1999.

16. A. Doucet, On Sequential Monte Carlo Methods for Bayesian Filtering, Technical Report, Department of Engineering, University of Cambridge, UK (1998).

17. A. F. M. Smith, A. E. Gelfand, Bayesian Statistics without Tears: a Sampling-Resampling Perspective, American Statistician, 46 (1992) 84-88.

18. M. Pitt, N. Shephard, Filtering via Simulation: Auxiliary Particle Filters, Journal of American Statistical Association, 94 (1999) 590-599.

19. A. Doucet, V. Tadic, Parameter Estimation in General State-space Models using Particle Methods, Annals of the Institute of Statistical Mathematics, 55 (2003) 409-422.

20. D. S. Achilias, C. Kiparissides, Development of a General Mathematical Framework for Modeling Diffusion Controlled Free-radical Polymerization Reactions, Macromolecules, 25 (1992) 3739-3750.

21. K. R. Muske, J. B. Rawlings, J. H. Lee, Receding Horizon Recursive State Estimation. In R. Berber (Eds.), Methods of Model Based Process Control, Kluwer Academic Publishers, The Netherlands, 1995. 


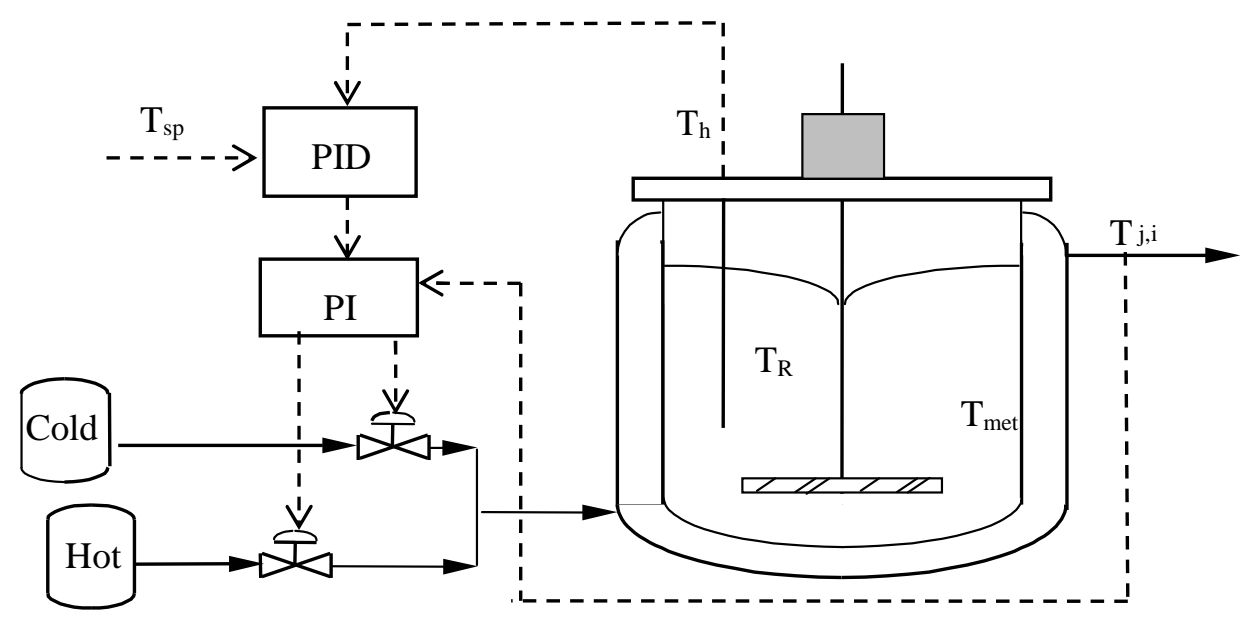

Figure 1. Batch polymerization reactor. 


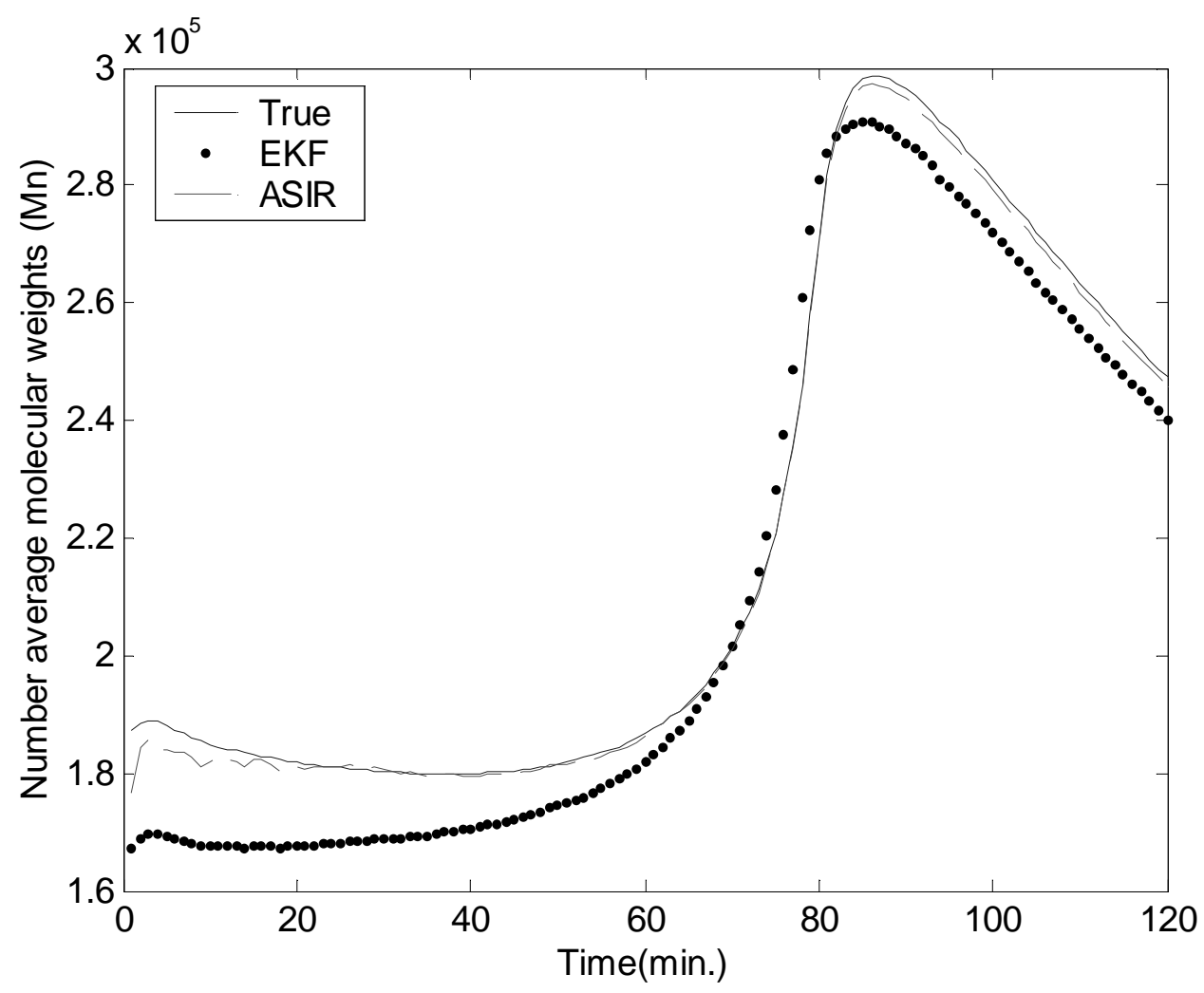

Figure 2. On-line estimation of number average molecular weights with unknown initiator concentration, with a $20 \%$ reduction in its nominal value. 200 particles were used for the ASIR filter. 


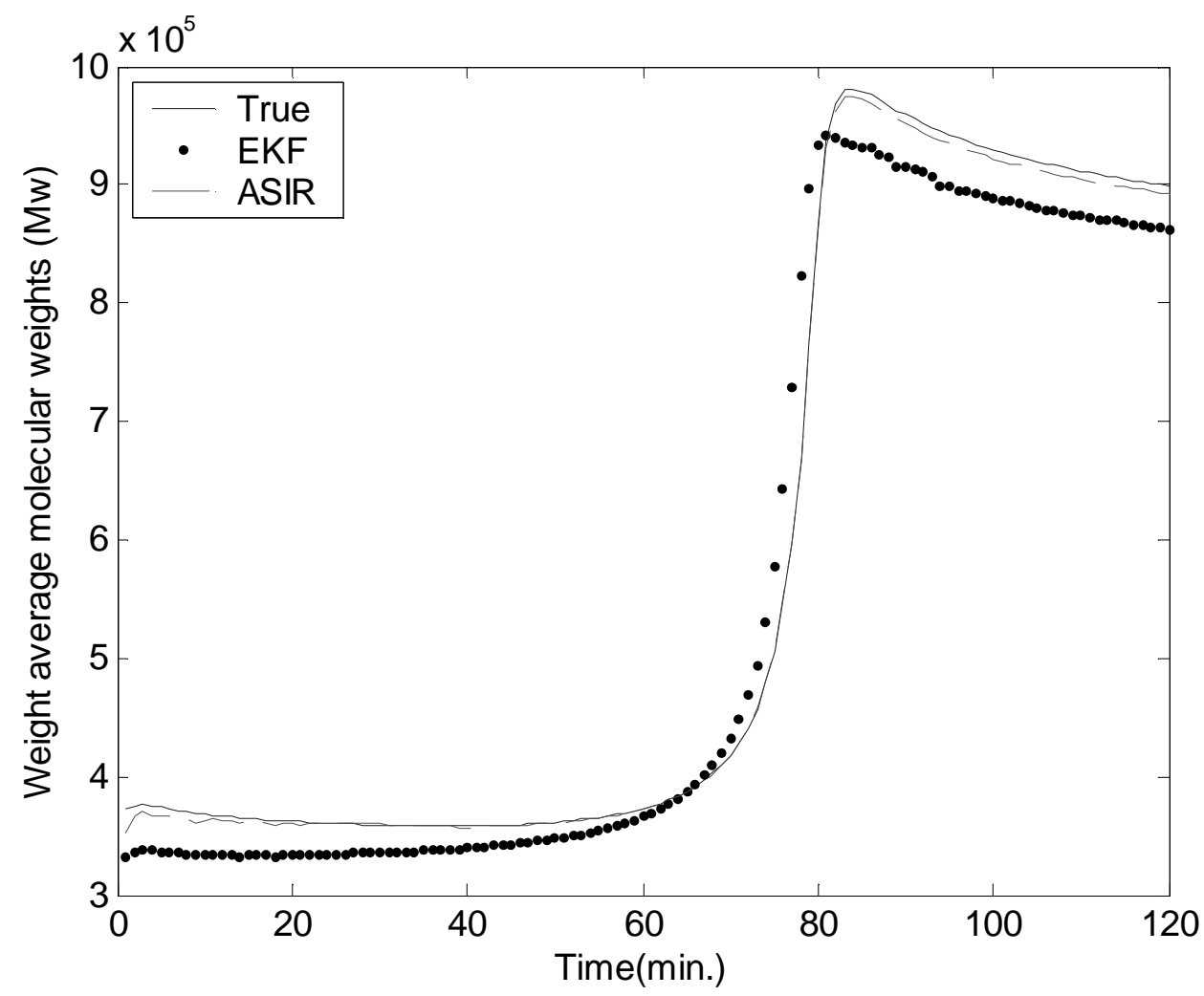

Figure 3. On-line estimation of weight average molecular weights with unknown initiator concentration, with a $20 \%$ reduction in its nominal value. 200 particles were used for the ASIR filter. 


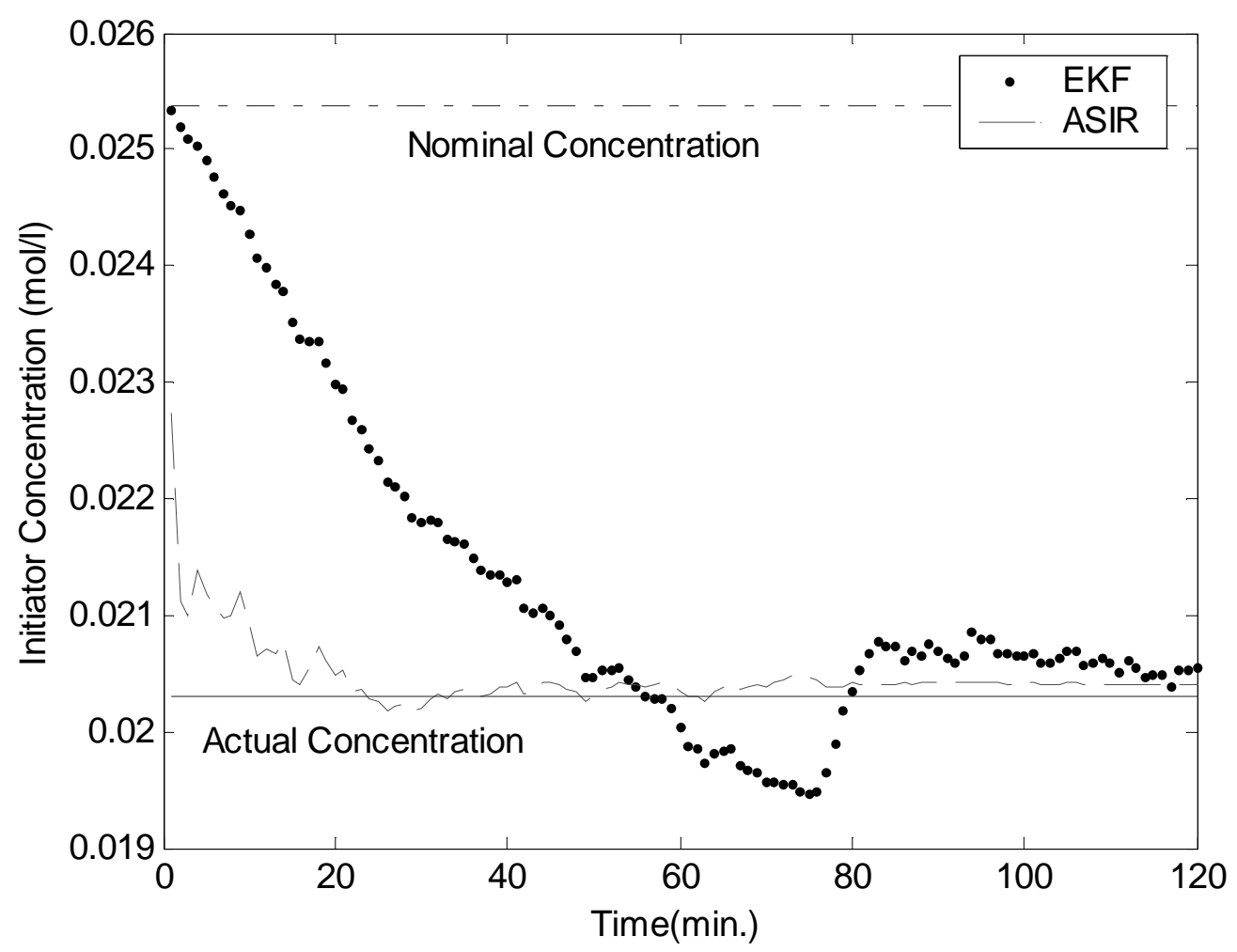

Figure 4. On-line estimation of initiator concentration, with a $20 \%$ reduction in its nominal value. 200 particles were used for the ASIR filter. 


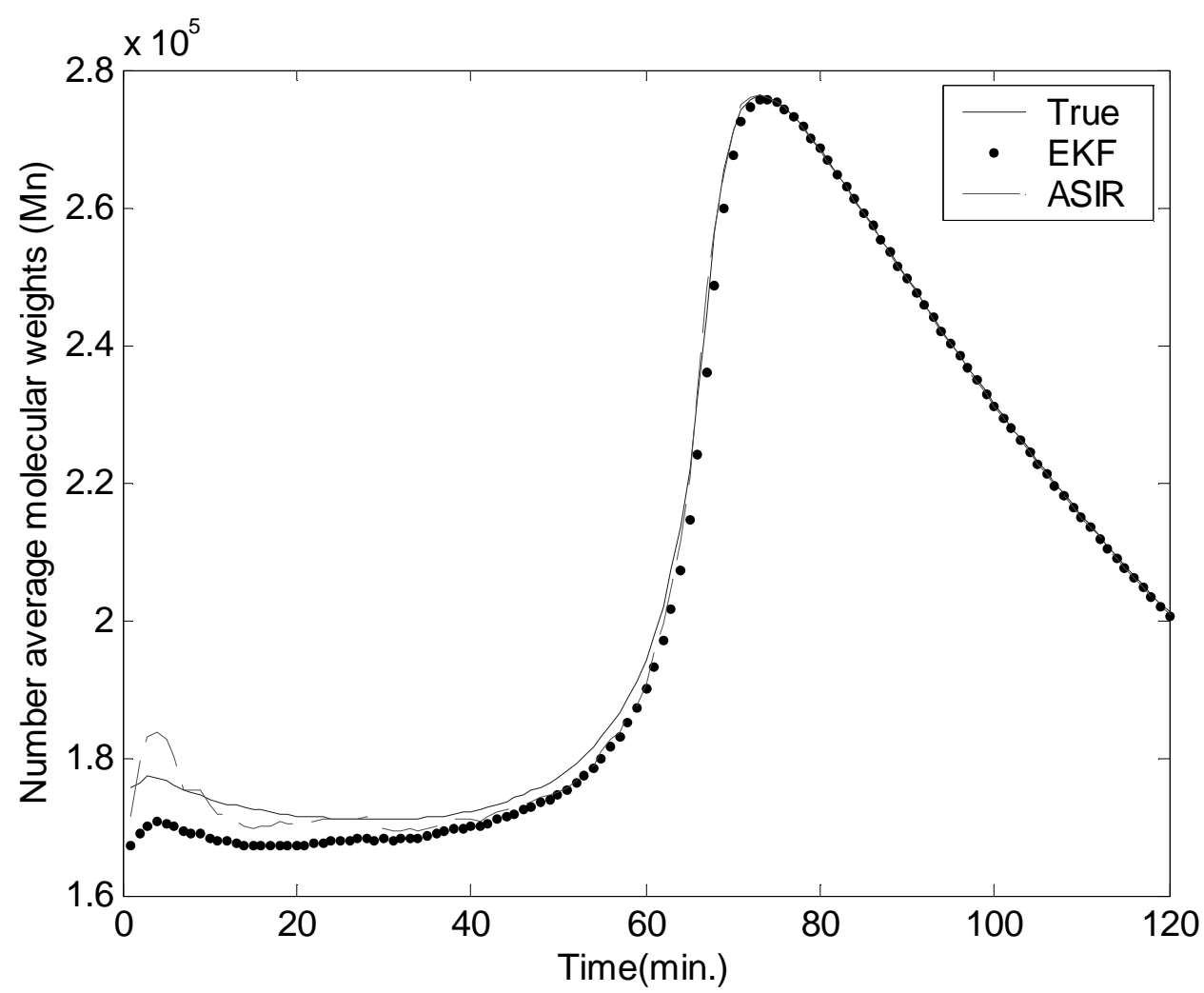

Figure 5. On-line estimation of number average molecular weights with unknown time-varying termination rate constant. 1000 particles were used for the ASIR filter. 


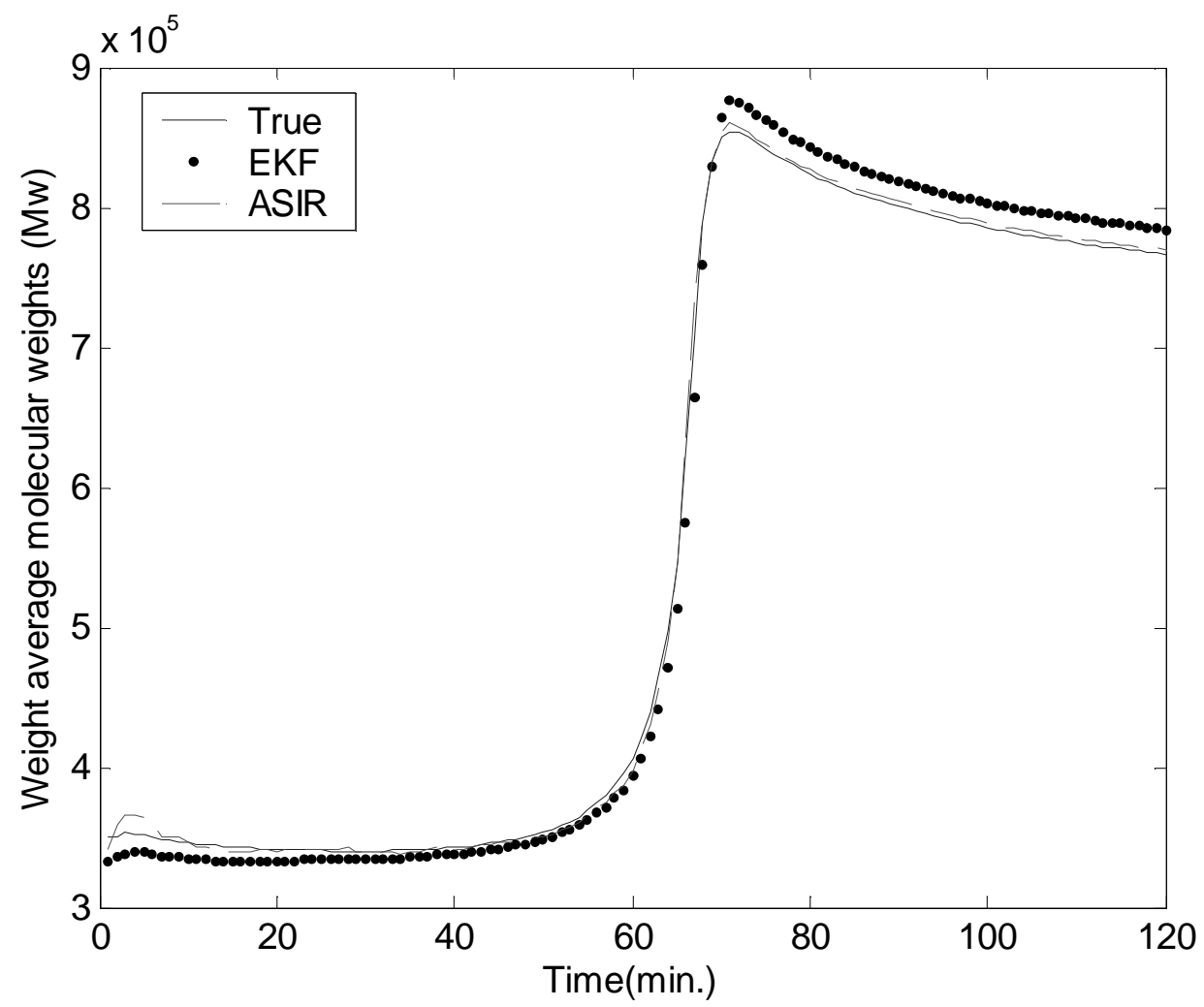

Figure 6. On-line estimation of weight average molecular weights with unknown time-varying termination rate constant. 1000 particles were used for the ASIR filter. 


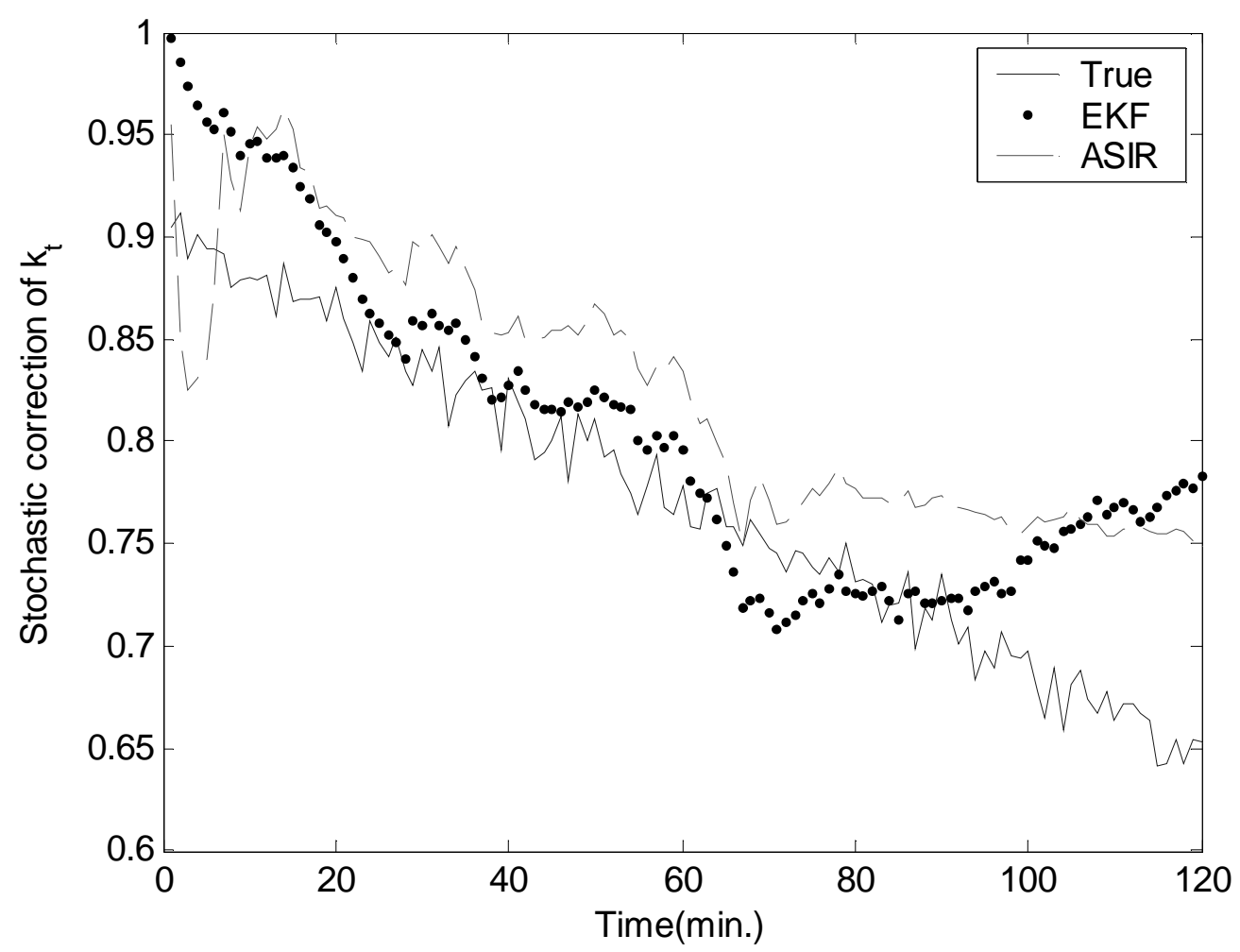

Figure 7. On-line estimation of the time-varying termination rate constant. 1000 particles were used for the ASIR filter. 
Table 1. System states

\begin{tabular}{|l|l|l|}
\hline State & Description & Initial value for simulation \\
\hline$x$ & Monomer conversion & 0 \\
\hline$\mu_{0}$ & Zero moment of dead polymer & 0 \\
\hline$\mu_{1}$ & First moment of dead polymer & 0 \\
\hline$\mu_{2}$ & Second moment of dead polymer & 0 \\
\hline$T_{R}$ & $\begin{array}{l}\text { Reactor or reacting mixture } \\
\text { temperature }\end{array}$ & $340{ }^{\circ} \mathrm{C}$ \\
\hline$T_{j, i}$ & $\begin{array}{l}\text {-th jacket part temperature } \\
(i=1,2,3,4)\end{array}$ & $340{ }^{\circ} \mathrm{C}$ \\
\hline$T_{m e t}$ & Metal wall temperature & $340{ }^{\circ} \mathrm{C}$ \\
\hline$T_{h}$ & Measured reactor temperature & $340{ }^{\circ} \mathrm{C}$ \\
\hline
\end{tabular}


Table 2. Estimation performance (RMSE) of average polymer molecular weight properties with unknown initiator concentration.

\begin{tabular}{|l|l|l|l|}
\hline Filters & EKF & ASIR $(N=200)$ & ASIR $(N=1000)$ \\
\hline$M n\left(\times 10^{3}\right)$ & $10.161 \pm 1.955$ & $2.561 \pm 1.629$ & $2.246 \pm 1.566$ \\
\hline$M w\left(\times 10^{3}\right)$ & $38.954 \pm 13.770$ & $9.606 \pm 9.136$ & $9.005 \pm 8.788$ \\
\hline CPU Time $(s)$ & $0.621 \pm 0.062$ & $6.786 \pm 0.378$ & $33.738 \pm 1.216$ \\
\hline
\end{tabular}


Table 3. Estimation performance (RMSE) of average polymer molecular weight properties with unknown time-varying termination rate constant.

\begin{tabular}{|c|c|c|}
\hline Filters & EKF & $\operatorname{ASIR}(N=1000)$ \\
\hline$M n\left(\times 10^{3}\right)$ & $4.461 \pm 1.714$ & $2.724 \pm 1.481$ \\
\hline$M w\left(\times 10^{3}\right)$ & $19.914 \pm 9.236$ & $10.126 \pm 8.115$ \\
\hline CPU Time $(s)$ & $0.718 \pm 0.058$ & $33.215 \pm 1.578$ \\
\hline
\end{tabular}

